\title{
THE ZERO-SETS OF THE RADIAL-LIMIT FUNCTIONS OF INNER FUNCTIONS
}

\author{
CHARLES L. BELNA, ROBERT D. BERMAN, PETER COLWELL, \\ AND GEORGE PIRANIAN
}

\begin{abstract}
A set $E$ on the unit circle is the zero-set of the radial-limit function of some inner function if and only if $E$ is a countable intersection of $F_{\sigma}$-sets of measure 0 .
\end{abstract}

\section{INTRODUCTION}

By Fatou's theorem [F] (or [CL, Theorem 2.1, p. 17]), the points $\zeta$ on the unit circle $C$ where the radial limit $f^{*}(\zeta)$ of a bounded holomorphic function $f$ in the unit disk $D$ exists constitute a set of Lebesgue measure $2 \pi$. If in addition $f \not \equiv w_{0}$, then the set $E\left(f, w_{0}\right)$ of points $\zeta$ on $C$ where $f^{*}(\zeta)=w_{0}$ has measure 0 , by a theorem of F. Riesz and M. Riesz [RR, p. 41] (or [CL, Theorem 2.5, p. 22]). On the other hand, to each set $E$ of measure 0 on $C$ correspond nonconstant, bounded holomorphic functions $f$ in $D$ such that the ordinary limit of $f$ (and therefore the radial limit of $f$ ) is 0 everywhere on $E$ (see [P, p. 214] or [Z, Vol. I, end of p. 276]).

G. T. Cargo has pointed out that not every set of measure 0 on $C$ is contained in the set $E(f, 0)$ of some inner function $f$, that is, of some bounded holomorphic function satisfying almost everywhere on $C$ the condition $\left|f^{*}(\zeta)\right|=1$. (We refer to $[\mathrm{CL}]$ for background concerning the class of inner functions and the subclasses of singular inner functions and Blaschke products that will be considered in the sequel.) Cargo showed [C, Theorems 1 and 4] that for each complex number $w_{0}$ and each nonconstant inner function $f$ the set $E\left(f, w_{0}\right)$ is meagre; in other words, it is a set of first category.

In [B], the second author considered the problem of characterizing the sets $E(f, 0)$ for inner functions $f$. He showed that if $f$ is an inner function, then the set of points where $f$ has the radial limit 0 is the intersection of countably many $F_{\sigma}$-sets of measure 0 . Theorem 2.4 in [B] gives some sufficient conditions for a set $E$ on $C$ to be the zero-set of the radial-limit function of an inner function. Example: if $E$ is the intersection of an $F_{\sigma}$-set of measure 0 with a $G_{\delta}$-set, then the zero-set of the radial-limit function of some Blaschke product is equal to $E$.

The present paper completes the characterization.

Received by the editors January 4, 1994 and, in revised form, November 11, 1994.

1991 Mathematics Subject Classification. Primary 30D40, 30D50.

Key words and phrases. Inner functions, radial-limit functions, zero sets. 
Theorem. In order that a set on the unit circle $C$ be the set where some inner function has the radial limit 0 , it is necessary and sufficient that it be a countable intersection of $F_{\sigma}$-sets of measure 0 .

Our proof of the sufficiency in the theorem requires a construction involving certain inner functions. In Section 2, we develop our elementary building blocks; in Section 3, we perform the final synthesis. Section 4 is devoted to some concluding remarks.

The authors gratefully acknowledge the referee's helpful suggestions.

\section{SINGULAR INNER FUNCTIONS}

We use functions of the form

$$
f(z)=\exp \left(\frac{1}{2 \pi} \int_{0}^{2 \pi} \frac{z+e^{i t}}{z-e^{i t}} d \mu(t)\right),
$$

where the symbol $\mu$ in the Stieltjes integral denotes a nondecreasing function that is defined on the real line and satisfies at each point $t$ the three conditions

$$
\begin{array}{ll}
\mu(0)=0 & \text { (initial standardization), } \\
\mu(t+2 \pi)-\mu(t)=\mu(2 \pi)=\|\mu\| & (2 \pi \text {-periodicity of the distribution } d \mu), \\
\mu\left(t^{+}\right)+\mu\left(t^{-}\right)=2 \mu(t) & \text { (normalization at discontinuities) } .
\end{array}
$$

At each point $z=r e^{i \theta}$ in $D$, the real part of $\log [1 / f(z)]$ is

$$
u(z)=\frac{1}{2 \pi} \int_{0}^{2 \pi} \frac{1-r^{2}}{(1-r)^{2}+4 r \sin ^{2}[(t-\theta) / 2]} d \mu(t) .
$$

For each fixed point $z=r e^{i \theta}(r>0)$ in $D$, the integrand in (2) is a decreasing function of $|t-\theta|(0 \leq|t-\theta| \leq \pi)$. This implies that if we interpret $d \mu$ as a distribution on the unit circle $C$ and shift all (or a portion) of the mass in $d \mu$ to a point nearer to $e^{i \theta}$, the change does not decrease the value of $u(z)$. Suppose, for example, that $\mu$ is constant on an arc

$$
A=\left\{\zeta: \zeta=e^{i t}, t_{1}<t<t_{2}\right\}
$$

and that the point $z$ lies in the sector $S$ of $D$ bounded by $A$ and by the two line segments joining the origin to the endpoints of $A$. To be definite, suppose that $\left|z-e^{i t_{1}}\right| \leq\left|z-e^{i t_{2}}\right|$. Shifting the entire mass of $d \mu$ to $e^{i t_{1}}$, we obtain a new function

and we deduce that

$$
\mu^{*}(t)= \begin{cases}0, & t_{1}-2 \pi<t<t_{1}, \\ \|\mu\|, & t_{1}<t<t_{1}+2 \pi,\end{cases}
$$

$$
\log \frac{1}{|f(z)|} \leq \frac{\|\mu\|}{2 \pi} \Re e \frac{e^{i t_{1}}+z}{e^{i t_{1}}-z} .
$$

The mapping $w(z)=\left(e^{i t_{1}}+z\right) /\left(e^{i t_{1}}-z\right)$ carries each circle $\Gamma$ internally tangent to $C$ at $e^{i t_{1}}$ onto a vertical line; moreover, if $\delta$ denotes the diameter of $\Gamma$, the image of $\Gamma$ passes through the point $w=(2-\delta) / \delta=2 / \delta-1$. Dealing similarly with points $z$ in the half of $S$ whose boundary passes through $e^{i t_{2}}$, we see that if $\mu$ is constant on $A$, the inequality

$$
\log \frac{1}{|f(z)|} \leq \frac{\|\mu\|}{2 \pi}\left(\frac{2}{\delta}-1\right)
$$


holds at each point $z$ in $S$ lying outside the union of the two disks of diameter $\delta$ that are internally tangent to $C$ at $e^{i t_{1}}$ and $e^{i t_{2}}$, respectively.

In connection with (1), we also recall that if the function $\mu$ in (1) has an infinite derivative at a point $t=\theta$, then, by virtue of a standard proof of Fatou's radial-limit theorem, the function $f$ has the radial limit 0 at $e^{i \theta}$.

In the sequel, we shall use a lemma that is proved in passing (but not stated) in the upper half of page 9 of [LP].

Lemma LP. If $E$ is a closed subset of $C$ of measure 0 , then there exists a function $\mu$ that meets the specifications stated in connection with the formula (1) and has the property

$$
\mu^{\prime}(t)= \begin{cases}+\infty, & e^{i t} \in E, \\ 0, & e^{i t} \in C \backslash E .\end{cases}
$$

To prove the lemma, we point out that if $E$ is a closed set of measure 0 in $C$, then each point of $E$ is either a bilateral limit point of $E$ or the endpoint of a component of $C \backslash E$. In the first case, the function $\mu$ constructed in the second paragraph of page 9 of [LP] has infinite right- and left-hand derivatives at $t$, and therefore $\mu^{\prime}(t)=\infty$. In the second case, $\mu$ has a saltus at $t$, and therefore $\mu^{\prime}(t)=\infty$ by virtue of the normalization.

Corresponding to each number $\delta$ in the interval $(0,2)$, let $T=T(E, \delta)$ denote the union of all circular disks that have diameter $\delta$ and are internally tangent to $C$ at some point of $E$. We note that the number of components of $T$ is less than $2 \pi / \delta$. In our application of Lemma LP to a function $f$ of the form (1), it will be useful to consider the auxiliary mental picture consisting of the graph W of the real-valued function $|f|$ over the unit disk $D$ in $z h$-space $(h \geq 0)$. The following informal proposition may be visualized in terms of the topography encountered by an imaginary traveler on the surface $W$.

Proposition. Let $f$ be a function given by (1), and denote by $W$ the surface described above. Then, for each point $z$ in $D \backslash T$, the point $(z,|f(z)|)$ on $W$ lies above the plane $h=\exp (-\|\mu\| / \delta \pi)$. If the function $\mu$ has an infinite derivative at the point $t$, then the closure of $D$ is (in a reasonable sense) tangent at $\left(e^{i t}, 0\right)$ to the image on $W$ of each triangular domain in $D$ that has a vertex at $e^{i t}$.

Consequently, any traveler on $W$ who strays onto the image of the territory $T$ incurs the risk of sliding to the bottom of a steep-walled pit.

A subset $E$ of $C$ satisfies the condition in our theorem if and only if it has a representation

$$
E=\bigcap_{j=1}^{\infty} E_{j},
$$

where each set $E_{j}$ is the union of closed sets $E_{j k}(k=1,2, \ldots)$ of measure 0 . Because every closed set of measure 0 on $C$ is nowhere dense, we can assume that for each index $j$ the sets $E_{j k}(k=1,2, \ldots)$ are pairwise disjoint ([S], see also [B, Theorem 3.2 and Corollary 3.4]). Also, since a point $e^{i t}$ lies in $E$ if and only if it lies in each of the sets $E_{j}(j=1,2, \ldots)$, and since, moreover, the intersection of two sets of type $F_{\sigma}$ is again of type $F_{\sigma}$, we can assume that the relation $E_{j} \supset E_{j+1}$ holds for each index $j$. Finally, to eliminate certain 
trivialities from our exposition, we assume that $E$ is nonempty and if one of the sets $E_{j k}$ is empty, then $E_{m n}$ is empty whenever $m=j$ and $n \geq k$.

Corresponding to each of our sets $E_{j}$, we shall construct a singular inner function $f_{j}$ such that $E\left(f_{j}, 0\right)=E_{j}$. The construction of $f_{j}$ will be strongly affected by a certain sequence $\left(\delta_{j k}\right)$ of positive numbers; our choice of each number $\delta_{1 k}$ will depend on the geometric relations among the sets $E_{1 m}$ ( $m \leq$ $k$ ); if $j>1$, the choice of $\delta_{j k}$ will reflect also the relations between the sets $E_{j-1}$ and $E_{j}$.

For the sake of notational consistency with what will follow, we introduce the alternate symbol $F_{1 k}$ for the set $E_{1 k}(k=1,2, \ldots)$. The intersections $E_{2 m} \cap F_{1 n}(m, n=1,2, \ldots)$ form a countable collection of disjoint closed sets whose union is the set $E_{2}$. We order the nonempty elements of this collection into a simple sequence $\left(F_{2 k}\right)(k=1,2, \ldots)$. More generally, once the sequence $\left(F_{j-1, k}\right)_{k=1}^{\infty}$ is defined, we order the family of all nonempty closed sets $E_{j m} \cap$ $F_{j-1, n}(m, n=1,2, \ldots)$ into a simple sequence $\left(F_{j k}\right)_{k=1}^{\infty}$ of disjoint closed sets.

For $\delta_{11}$ we choose any number in the interval $(0,1)$, and we construct the corresponding territory $T_{11}=T\left(F_{11}, \delta_{11}\right)$. After choosing constants $\delta_{1 m}$ and constructing the corresponding territories $T_{1 m}=T\left(F_{1 m}, \delta_{1 m}\right)(m=1, \ldots, j-$ $1)$, we choose a number $\delta_{1 j}$ lying in $\left(0, \delta_{11}\right)$ and small enough so that the territory $T_{1 j}=T\left(F_{1 j}, \delta_{1 j}\right)$ lies at a positive distance from each of the territories $T\left(E_{1 m}, \delta_{1 m}\right)(m<j)$.

Suppose we have chosen the numbers $\delta_{m \nu}$ for $m=1, \ldots, j-1$ and $\nu=$ $1,2, \ldots$ and for $m=j$ and $\nu<k$. Let $n$ be the unique positive integer for which the set $F_{j k}$ is a subset of $F_{j-1, n}(n=1,2, \ldots)$. Clearly, we can choose $\delta_{j k}$ small enough so that the territory $T_{j k}=T\left(F_{j k}, \delta_{j k}\right)$ is a subset of $T_{j-1, n}$ and lies at a positive distance from each of the territories $T_{j h}(h=$ $1,2, \ldots, k-1)$.

Corresponding to each index $j$, we denote the union of the territories $T_{j k}$ $(k=1,2, \ldots)$ by $T_{j}$. It is easy to see that if $e^{i t} \in C \backslash E_{j}$ and the radius $R_{t}=\left[0, e^{i t}\right)$ meets a component of a territory $T_{j k}$, then the endpoints of the segment $R_{t} \cap T_{j k}$ lie in none of the territories $T_{j h}(h \neq k)$ and in none of the sets $T_{j+1}, T_{j+2}, \ldots$.

If $c_{j k}$ is a positive constant and $\mu_{j k}^{*}$ is a function satisfying with regard to the set $F_{j k}$ the condition described in Lemma LP, then the function $c_{j k} \mu_{j k}^{*}=\mu_{j k}$ also satisfies that condition. By virtue of the inequality (3), we can choose the constant $c_{j k}$ small enough so that the function $f_{j k}$ generated by $\mu_{j k}$ in accordance with the formula (1) has at each point of $F_{j k}$ the radial limit 0 and satisfies everywhere in $D \backslash T_{j k}$ the inequality

$$
\log \frac{1}{\left|f_{j k}(z)\right|}<\frac{1}{2^{j+k}}
$$

If $e^{i t} \in F_{j k}$, then $f_{j k}$ has at $e^{i t}$ the radial limit 0 ; if $e^{i t} \in C \backslash F_{j k}$, then the function $\mu_{j k}$ is constant on some open segment with the midpoint $t$, and therefore $f_{j k}$ is holomorphic at $e^{i t}$ and $\left|f_{j k}\left(e^{i t}\right)\right|=1$. Clearly, the function $\mu_{j}=\sum_{k} \mu_{j k}$ has finite norm, and by a classical differentiation theorem of 
Fubini, $\mu_{j}^{\prime}=0$ a.e. Also, the product

$$
f_{j}=\prod_{k=1}^{\infty} f_{j k}
$$

converges uniformly on compact sets, satisfies everywhere in $D \backslash T_{j}$ the inequality

$$
\log \frac{1}{\left|f_{j}(z)\right|}<\frac{1}{2^{j}}
$$

and has at each point of $E_{j}$ the radial limit 0 .

Suppose now that $e^{i t} \in C \backslash E_{j}$. Because $\delta_{j k} \rightarrow 0$ as $k \rightarrow \infty$ and

$$
\operatorname{dist}\left(T_{j h}, T_{j k}\right)>0
$$

whenever $h \neq k$, each of the radial segments

$$
\left\{z: z=r e^{i t}, 1-1 / m \leq r<1\right\}
$$

contains a point $z_{m}$ in $D \backslash T_{j}$. The inequality (4) implies that the relation

$$
\prod_{k>K}\left|f_{j k}\left(z_{m}\right)\right|>\exp \left(-2^{-j-K}\right)
$$

holds for all positive integers $K$ and $m$. Since the right-hand side tends to 1 as $K \rightarrow \infty$, and since each of the $K$ factors $f_{j 1}, \ldots, f_{j K}$ is holomorphic and has modulus 1 at $e^{i t}$, it follows that the sluster set at $e^{i t}$ of $f_{j}$ contains at least one point of modulus 1 .

\section{Celebration after the grubby wORK}

It remains only to combine the functions $f_{j}$ into a function $f$ whose radial limit at each point of the set $E=\bigcap E_{j}$ is 0 and whose radial cluster set at each of the points of $C \backslash E$ includes a point other than 0 .

Corresponding to each index $j$, let

$$
g_{j}=\frac{1 / 2+f_{j}}{1+f_{j} / 2}
$$

Clearly, $g_{j}$ is an inner function. At each point where the radial limit of $f_{j}$ is 0 , the radial limit of $g_{j}$ is $1 / 2$, and at each point where the radial cluster set of $f_{j}$ includes a point of $C$, the radial cluster set of $g_{j}$ also includes a point of $C$. Moreover, because $-1 / 2$ is not the radial limit of $f_{j}$ at any point of $C$, the function $g_{j}$ does not have the radial limit 0 at any point, and therefore $g_{j}$ has no singular factor; in other words, $g_{j}$ is a Blaschke product, that is, a function of the form

$$
B_{j}(z)=e^{i \gamma_{j}} z^{\nu_{j}} \prod_{n=1}^{\infty}\left|a_{j n}\right| \frac{1-z / a_{j n}}{1-\bar{a}_{j n} z},
$$

where $\gamma_{j}$ and $\nu_{j}$ represent a real constant and a nonnegative integer, respectively, and where the points $a_{j n}$ lie in $D \backslash\{0\}$ and satisfy the condition $\sum_{n}\left(1-\left|a_{j n}\right|\right)<\infty$.

Except in the trivial case where $E=\varnothing$, formula (1) guarantees that the value of $\log 1 / f_{j}(0)$ has a positive value, and therefore $B_{j}(0)$ is positive. This in turn 
implies that the factors $e^{i \gamma_{j}}$ and $\nu_{j}$ in our formula for $B_{j}(z)$ have the values 1 and 0 , respectively.

The final stage of our synthesis is the creation of the formal product $f=$ $\prod g_{j}$. That the symbol $f$ represents a genuine Blaschke product is equivalent to the convergence of the double series

$$
\sum_{j=1}^{\infty} \sum_{n=1}^{\infty}\left(1-\left|a_{j n}\right|\right)
$$

This follows from the inequality $\log r<r-1(r>0)$, the equality $1 / B_{j}(0)=$ $\prod_{n=1}^{\infty}\left|a_{j n}\right|^{-1}$, and the absolute convergence of the series $\sum_{j=1}^{\infty} \log 1 / B_{j}(0)$.

To see that the Blaschke product $f$ has the desired property, suppose first that $e^{i t} \in E$. Then $e^{i t} \in E_{j}(j=1,2, \ldots)$, and because each of the functions $g_{j}$ has at $e^{i t}$ the radial limit $1 / 2$, the radial limit of $f$ is 0 , at the point $e^{i t}$.

Next, suppose that $e^{i t} \in C \backslash E$, and let $J$ denote the least nonnegative integer such that $e^{i t} \in C \backslash E_{J+1}$. At the point $e^{i t}$, the product of the first $J$ factors $g_{j}$ has the radial limit $2^{-J}$. As we pointed out in Section 2, the radius $\left[0, e^{i t}\right)$ supports a sequence $\left\{z_{m}\right\}$ that converges to $e^{i t}$ and lies in $D \backslash T_{J+1}$. Because the sequence of sets $T_{j}$ decreases, the sequence $\left\{z_{m}\right\}$ lies in each of the sets $D \backslash T_{j}(j=J+1, J+2, \ldots)$. To study the behavior of the corresponding factors $g_{j}(j=J+1, J+2, \ldots)$ on the sequence of points $z_{m}(m=1,2, \ldots)$, we point out that if $\left|f_{j}\left(z_{m}\right)\right|=\rho$, then, by virtue of formula (5), the function $g_{j}$ carries the point $z_{m}$ to a point on the circle having a diameter with the endpoints

$$
\frac{1-2 \rho}{2-\rho} \text { and } \quad \frac{1+2 \rho}{2+\rho}
$$

on the real line. Because the left-hand endpoint is nearer to the origin than the one on the right,

$$
\left|g_{j}\left(z_{m}\right)\right| \geq\left|\frac{1-2 \rho}{2-\rho}\right| \text {. }
$$

The inequality $\left|f_{j}\left(z_{m}\right)\right|>1 / 2$ resolves the ambiguity of sign, and we see that

$$
1-\left|g_{j}\left(z_{m}\right)\right| \leq 1-\frac{2 \rho-1}{2-\rho}=\frac{3(1-\rho)}{2-\rho}<3\left(1-\left|f_{j}\left(z_{m}\right)\right|\right)
$$

Since the values of $g_{j}\left(z_{m}\right)$ are bounded away from zero, there exists a constant $c$ such that

$$
-\log \left|g_{j}\left(z_{m}\right)\right|<c\left(1-\left|g_{j}\left(z_{m}\right)\right|\right)<3 c\left(1-\left|f_{j}\left(z_{m}\right)\right|\right) .
$$

It follows that

$$
\log \prod_{j>J} \frac{1}{\left|g_{j}\left(z_{m}\right)\right|}<3 c \sum_{j>J}\left(1-\left|f_{j}\left(z_{m}\right)\right|\right) .
$$

The inequality (4) implies that to each positive number $\epsilon$ there corresponds an integer $N(N \geq J)$ such that the inequality

$$
\sum_{j>N}\left(1-\left|f_{j}\left(z_{m}\right)\right|\right)<\epsilon
$$

holds for each index $m$. The argument at the end of Section 2 shows that for all sufficiently large values of the index $m$, the number $\epsilon$ is also an upper bound for the sum of the analogous terms with the indices $j=J+1, \ldots, N$. 
It follows that the radial cluster set at $e^{i t}$ of the product $\prod_{j>J} g_{j}\left(z_{m}\right)$ contains at least one point of $C$, and we see immediately that the radial cluster set at $e^{i t}$ of the function $f$ contains at least one point of the circle $|w|=2^{-J}$. This concludes the proof that every subset of $C$ that is a countable intersection of $F_{\sigma}$-sets of measure 0 is the set where an inner function has radial limit 0 .

We point out that if some open subset $A$ of $C$ contains no points of the set $E$, then each component of $A$ lies in a domain (cuspidate at both ends of the component) that contains no zeros of any of the Blaschke products $g_{j}$. Consequently, the Blaschke product $f$ is holomorphic and has absolute value 1 on each component of $A$. Moreover, corresponding to each positive $\epsilon$ and each compact subset $K$ of $D \cup A$, we can choose our set $\left\{\delta_{j k}\right\}(j, k=1,2, \ldots)$ so that the inequality $|f(z)-1|<\epsilon$ holds everywhere in $K$.

\section{The CASES WHERE $w \neq 0$}

The following extension of our main theorem holds.

If $w \in D \backslash\{0\}$, then a set $E$ on $C$ is the set $E\left(f_{w}, w\right)$ for some Blaschke product $f_{w}$ if and only if $E$ is a countable intersection of $F_{\sigma}$-sets of measure 0 .

The necessity of the condition is obvious. To prove sufficiency, suppose first that $|w| \neq 2^{-1}, 2^{-2}, 2^{-3}, \ldots$. We construct the function $f$ as in Sections 2 and 3 and define $f_{w}$ by the formula

$$
f_{w}=\frac{w+f}{1+\bar{w} f}
$$

Then $f_{w}$ has the radial limit $w$ at all points $e^{i t}$ where $f$ has the radial limit 0 . Moreover, since $-w$ is not a radial limit value for $f$, the set $E\left(f_{w}, 0\right)$ is empty, and therefore $f_{w}$ is a Blaschke product.

If $|w|$ has one of the values $2^{-m}(m=1,2, \ldots)$, we proceed in the same way, except that we now define the Blaschke products $B_{j}$ by the formula

$$
B_{j}=\frac{1 / 3+f_{j}}{1+f_{j} / 3} \text {. }
$$

In the case where $f$ is an inner function and $|w|=1$, the set $E(f, w)$ is still of type $F_{\sigma \delta}$; also, the theorems of F. and M. Riesz and of Cargo mentioned in our introduction are still applicable. But it has been shown (see [BN]) that $E(f, w)$ need not be contained in an $F_{\sigma}$-set of measure 0 . In other words, the characterization of the sets $E(f, w)$ for inner functions depends on whether $w \in D$ or $w \in C$.

An interesting question-perhaps easier than the problem of complete characterization of the sets $E(f, 1)$-is whether every meagre $F_{\sigma \delta}$-set of measure 0 is contained in the set $E(f, 1)$ for some nonconstant inner function $f$.

Another natural extension of the radial-limit zero-set problem concerns the possibility of radial-limit interpolation. We refer to [BN] for results in this direction and a discussion of the literature.

\section{REFERENCES}

[B] R. D. Berman, The sets of fixed radial limit value for inner functions, Illinois J. Math. 29 (1985), 191-219.

[BN] R. D. Berman and T. Nishiura, Some mapping properties of the radial-limit function of an inner function, J. London Math. Soc. (to appear). 
[C] G. T. Cargo, Some topological analogues of the F. and M. Riesz uniqueness theorem, J. London Math. Soc. (2) 12 (1975/76), 64-74.

[CL] E. F. Collingwood and A. J. Lohwater, The theory of cluster sets, University Press, Cambridge, 1966.

[F] P. Fatou, Séries trigonométriques et séries de Taylor, Acta Math. 30 (1906), 335-400.

[LP] A. J. Lohwater and G. Piranian, The boundary behavior of functions analytic in a disk, Ann. Acad. Sci. Fenn. Ser. Math. 239 (1957).

[P] I. I. Priwalow, Randeigenschaften analytischer Funktionen, VEB Deutscher Verlag der Wissenschaften, Berlin, 1956.

[RR] F. Riesz and M. Riesz, Über die Randwerte einer analytischen Funktion, Quatrième Congrès des Math. Scand., 1916, pp. 27-44.

[S] W. Sierpiński, Sur une classification des ensembles mesurables (B), Fund. Math. 10 (1927), 320-327.

[Z] A. Zygmund, Trigonometric series, 2nd ed., Cambridge University Press, London and New York, 1968.

342 OAK Street, Lakeside, Ohio 43440

Department of Mathematics, Wayne State University, Detroit, Michigan 48202

E-mail address: rberman@math.wayne.edu

Department of Mathematics, Iowa State University, Ames, Iowa 50011

Department of Mathematics, University of Michigan, AnN Arbor, Michigan 481091003 